\title{
Zur Methode der enzymatischen Neutralfett-Bestimmung in biologischem Material
}

\author{
Von F. H. SCHMIdT und K. von DaHL \\ Aus der Pbysiol.-Chem. Abteilung der Forscbungslaboratorien der C. F. Boebringer \& Soebne GmbH, Mannbeim
}

(Eingegangen am 13. Februar 1968)

Die alkalische Hydrolyse des Neutralfetts führt zur quantitativen Freisetzung von Glycerin, das im zusammengesetzten optischen Test mittels Glycerokinase bestimmbar ist. Eine quantitative Bestimmung in biologischem Material ist an die folgenden Voraussetzungen geknüpft:

1. Beseitigung von fettsäurebedingten Trübungen durch Magnesiumsalzfällung.

2. Korrektur der gemessenen Extinktionen durch regelmäßige Verwendung von Reagenzien-Leeransätzen.

3. Verwendung von glycerinfreier $\mathrm{KOH}$.

4. Beseitigung des durch Glucose bedingten Fehlers (wichtig für die Bestimmung des freien Glycerins).

5. Die Konzentration an freiem Glycerin im Serum ist gering und kann für die Praxis bei der Neutralfett-Bestimmung vernachlässigt werden.

Es wird eine Modifikation der enzymatischen Glycerid-Bestimmung in biologischen Flüssigkeiten beschrieben, die auch für Gewebe anwendbar und frei von den genannten Störmöglichkeiten ist.

The alkaline hydrolysis of neutral fat gives a quantitative yield of glycerol, which can be determined by the coupled enzyme-optical test with glycerokinase. The quantitative determination in biological material is possible providing the following conditions are observed:

1. Turbidity caused by fatty acids is removed by precipitation with a magnesium salt.

2. The measured extinction is always corrected by the use of a reagent blank.

3. Glycerol-free $\mathrm{KOH}$ is used.

4. The error caused by glucose (important in the determination of free glycerol) is removed.

5. The concentration of free glycerol in the serum is low and, for practical purposes, can be ignored in the determination of neutral fat.

A modification of the enzymatic determination of glycerides in biological fluids is described; it can also be used for tissues, and it is free from the above sources of error.

Die Bestimmung der Triglyceride im Serum oder auch in anderen biologischen Flüssigkeiten führt in der Regel über die Hydrolyse des Neutralfetts zur quantitativen Freisetzung von Glycerin, das enzymatisch meßbar ist. Für diese Bestimmung kann sowohl nach Wieland (1) mit Hilfe von Glycerokinase ${ }^{1}$ ) und Glycerophosphatdehydrogenase verfahren werden oder aber man verwendet das von KREUTZ (2) bzw. KREUTZ und EgGStein (3) mitgeteilte Verfahren der kombinierten Anwendung von Glycerokinase, Pyruvatkinase und Lactatdehydrogenase. Die Bestimmung basiert auf der folgenden Reaktion:

Triglyceride $\stackrel{\text { äthanol. } \mathrm{KOH}}{\rightarrow}$ Glycerin + Fettsäuren Glycerin + ATP $\frac{\mathrm{GK}}{\left(\mathrm{Ng}^{++}\right)} \rightarrow$ Glycerophosphat-3 + ADP

$\mathrm{ADP}+\mathrm{PEP} \underset{\mathrm{Mg}^{++}}{\longrightarrow} \mathrm{ATP}+$ Pyruvat

Pyruvat $+\mathrm{NADH}+\mathrm{H}^{+} \stackrel{\mathrm{LDH}}{\longrightarrow}$ Lactat $+\mathrm{NAD}^{+}$.

Das Verfahren, biologisches Material direkt der Hydrolyse zu unterwerfen, hat sich nunmehr weitgehend durchgesetzt, wenngleich durchaus im Einzelfall Überlegungen dafür sprechen, mit Extrakten zu arbeiten.

1) Abkiirzingen: $\mathrm{GK}=$ Glycerokinase = ATP: Glycerin Phosphotransferase (EC 2.7.1.30); Glycerophosphatdehydrogenase = L-Glycerin-3-phosphat: NAD Oxydoreduktase (EC 1.1.1.8); PK $=$ Pyruvatkinase $=$ ATP: Pyruvat Phosphotransferase (EC 2.7.1.40) $; \mathrm{LDH}=$ Lactatdehydrogenese $=\mathrm{L}$-Lactat: NAD Oxydoreduktase (EC 1.1.1.27);] PEP = Phosphoenolpyruvat, $\mathrm{TRA}=$ Triäthanolamin. Alle verwendeten Substrate und Enzyme sind Produkte des Biochemica-Programms von Boehringer, Mannheim.
In einer Vielzahl von vergleichenden Untersuchungen hat sich uns die Extraktion nach FolCH-SPERRY (4) besonders bewährt. Von einem Extraktionsansatz von $0,2 \mathrm{~m} l$ Serum auf $5 \mathrm{~m} l$. FOLCH-SpERRY-Gemisch ${ }^{2}$ ) benötigt man nur $2 \mathrm{~m} l$ Extrakt $(0,08 \mathrm{~m} l$ Serum). Die Vorteile gegenüber dem Direktverfahren sind die folgenden:

1. Es werden für einen solchen Ansatz geringere Mengen Alkali für die Verseifung benötigt; hierdurch wird die Neutralisation mit $\mathrm{HClO}_{4}$ überflüssig.

2. Bei der Hydrolyse wird mit klaren Lösungen gearbeitet, wodurch Trübungen, Ausfällungen (Gefahr des Siedeverzugs!) vermieden werden.

3. Stillstand der Reaktion nach Glycerokinase-Zugabe in $1-2$ Min. Extrapolieren zur Ermittlung von $\Delta \mathrm{E}$ entfällt.

4. Es besteht die Möglichkeit, in aliquoten Teilen des Extraktes weitere klinisch-chemisch interessierende Lipide zu bestimmen. Außerdem kann der Extrakt ohne Gefahr der bakteriellen Zersetzung aufbewahrt werden. 5. Für die Untersuchung von Gewebeproben, insbesondere Biopsie-Material - hierüber wird an anderer Stelle berichtet (5) - empfiehlt sich das Extraktionsverfahren in jedem Fall. Die unter Störungen beschriebenen Modifikationen (s. dort) gelten für diese Aufarbeitung in gleicher Weise.

Die Anwendung der Direkthydrolyse im Serum macht es erforderlich, vor Durchführung des Testes den pH-Wert mittels Tüpfelung (a) - ein zeitaufwendiger

2) Chloroform-Methanol 2:1 $\mathrm{v} / \mathrm{v}$. 
Vorgang - exakt einzustellen, um Trübungen (b) durch das Auftreten von schwerlöslichen MagnesiumSalzen bzw. das Ausfällen von denaturierten Proteinen hintanzuhalten. Im Routinebetrieb ist dies nur schwierig verifizierbar, so daß es häufig zu Trübungen kommt, die durch den Magnesium-Zusatz im Test weiterhin begünstigt werden. Von den Autoren wird keine Blind-Reaktion (c) verlangt, um Spuren von Glycerin in der Glycerokinase und anderen Ingredienzien des Tests zu erfassen. Die Qualität der $\mathrm{KOH}$ (d) kann zu Fehlern führen, worauf nicht hingewiesen wird. Schließlich kann ein zu hoher Wert noch dadurch vorgetäuscht werden, daß die in der Glycerokinase in Spuren vorhandene Hexokinase Glucose zu Glucose-6phosphat umsetzt, wobei durch das entstandene ADP eine Phosphoenolpyruvat-verbrauchende Reaktion in Gang gesetzt wird. Es ergibt sich hierdurch ein kontinuierlicher "Schleich", der besonders bei der Bestimmung des freien Glycerins in Erscheinung tritt (e). Die in der Literatur mitgeteilten hohen Werte für freies Glycerin im Serum könnten mindestens teilweise hierdurch erklärt werden. $\mathrm{Ob}$ bei der Neutralfett-Bestimmung in jedem Fall das freie Glycerin parallel bestimmt werden muß, hängt von der Natur des Untersuchungsmaterials $a b(f)$.

Wir schließen durch die im folgenden beschriebenen Modifikationen die genannten Fehlermöglichkeiten (a-f) aus, wodurch das Ergebnis reproduzierbar (s. Tab. 4) und die Durchführung der Analyse vereinfacht wird.

\section{Magnesium-Fällung (Störung $\mathrm{a}+\mathrm{b}$ )}

Nach Beendigung der Hydrolyse werden $1,5 \mathrm{ml}$ 0,1 M $\mathrm{MgSO}_{4}$-Lösung dem Hydrolysat zugesetzt. Hierdurch wird der stark alkalische $\mathrm{pH}$-Wert auf $\sim 9-10 \mathrm{ab}-$ gestumpft und die freigesetzten Fettsäuren in Form von $\mathrm{Mg}$-Salzen niedergeschlagen, Tüpfelung ist nicht mehr erforderlich, Trübung wird hierdurch eliminiert.

Tab. 1

Fehlermöglichkeit durch Vernachlässigung von Blindwerten (Glycerin in Glycerokinase und sekundäre Glycerin-Kontamination)

\begin{tabular}{cccccc}
\hline Probe & $\begin{array}{c}\Delta \mathrm{E} \\
\text { (nicht } \\
\text { korrig.) }\end{array}$ & $\begin{array}{c}\mathrm{mg} / 100 \mathrm{ml} \\
\text { Triglyceride } \\
\text { (nicht } \\
\text { korrig.) }\end{array}$ & $\begin{array}{c}\Delta \mathrm{E} \\
\text { (korrig.) }\end{array}$ & $\begin{array}{c}\text { mg/100 ml } \\
\text { Triglyceride } \\
\text { (korrig.) }\end{array}$ & $\%$ Fehler \\
\hline 1 & 0,060 & 72 & 0,040 & 48 & 33 \\
2 & 0,090 & 109 & 0,070 & 84 & 23 \\
3 & 0,180 & 217 & 0,160 & 193 & 11 \\
\hline
\end{tabular}

\section{Blindwert (Störung $c$ )}

Nichtbeachten des Blindwerts kann' (s. Tab. 1) etwa $10-30 \%$ Fehler - bei Untersuchung von Leberzylindern bis $100 \%$ - bedingen. Das Ausmaß des Fehlers hängt naturgemäß vom Glycerin- bzw. FettGehalt des Analysenmaterials ab. Da die Blindextinktion Schwankungen unterliegt, ist für jede Versuchsreihe ein Blindwert mitzubestimmen, die Blindextinktion von der Extinktion des Hauptwerts abzuziehen.

\section{Glycerinfreie $K O H$ (Störung d)}

Für die Herstellung der äthanol. KOH ist auf glycerinfreie Qualität der Kalilauge zu achten (p. a.-Qualität in Form von Rotulis enthält unterschiedliche Mengen Glycerin, s. Tab. 2). Der Fehler kann bei einem Triglyceridspiegel von $100 \mathrm{mg} / 100 \mathrm{~m} l$ bis $87 \%$ ausmachen. Wir verwenden konzentrierte KOH-Lösung ${ }^{1}$ ) bzw. technische $\mathrm{KOH}^{2}$ ).

Tab. 2

Fehlermöglichkeiten durch unterschiedlichen Glyceringehalt der verwendeten KOH-Plätzchen*)

\begin{tabular}{lllllll}
\hline Plätzchen- No. & 1 & 2 & 3 & 4 & 5 & 6 \\
\hline $\begin{array}{l}\text { Glycerin } \\
\text { mg/100 g }\end{array}$ & 13 & 58 & 11 & 67 & 23 & 7 \\
$\begin{array}{l}\text { Rotuli } \\
\begin{array}{l}\text { 10 mg KOH } \\
\text { entspricht } \mu \mathrm{g}\end{array}\end{array}$ & & & & & \\
$\begin{array}{l}\text { Glycerin/Test } \\
\text { 1,3 }\end{array}$ & 5,8 & 1,1 & 6,7 & 2,3 & 0,7 \\
result. $\Delta \mathrm{E}$ & 0,015 & 0,065 & 0,012 & 0,076 & 0,026 & 0,008 \\
\hline
\end{tabular}

Einem Triglyceridspiegel von $100 \mathrm{mg} / 100 \mathrm{ml}$ (normal) entspricht

$\triangle \mathrm{E}$ von 0,087 .
Diese Verunreinigungen ergeben dann folgende Fehler in \%:

\begin{tabular}{lllllll}
\hline$\%$ Fehler & 17 & 75 & 14 & 87 & 30 & 9 \\
\hline
\end{tabular}

*) Kaliumhydroxyd-Plätzchen p. a. Merck (Charge 416655 ).

\section{Febler durch Glucose (Störung e)}

Dieser Fehler tritt nur bei Bestimmung des freien Glycerins in Erscheinung, ist jedoch bei der.Erfassung der Triglyceride zu vernachlässigen, da Glucose beim Hydrolyse-Vorgang durch die kochende äthanol. Kalilauge zerstört wird.

\section{Febler durch freies Glycerin (Störung $\mathrm{f}$ )}

Im Blutserum des Menschen - auch bei lipämischen Proben - ist unter Berücksichtigung des unter Störung Gesagten die Konzentration an freiem Glycerin sehr niedrig. Wir fanden bei unseren Untersuchungen Durchschnittswerte von $1 \mathrm{mg} / 100 \mathrm{ml}$, das entspricht unter Berücksichtigung des durchschnittlichen Molekulargewichts von 850 für Fett $9,2 \mathrm{mg} / 100 \mathrm{~m} l$ Triglycerid. Für die Belange der Praxis kann in der Regel auf die parallele Bestimmung des freien Glycerins verzichtet werden, doch sollte bei wissenschaftlichen Fragestellungen das Gesamtglycerid durch Erfassen des freien Glycerins korrigiert werden.

Der methodische Fehler kann - wie gezeigt wurde vor allem bei Nichtbeachten der Punkte 1-3 bei der Triglycerid-Bestimmung erheblich sein; er wird sich bei Fehlen aller Voraussetzungen darüber hinaus addieren, da die Störungen in der Regel zu falschpositiven Werten führen.

Nachstehend wird die Durchführung eines Testansatzes zur Triglycerid-Bestimmung, wie er. von uns verwendet wird, beschrieben. Mit diesem Vorgehen haben wir bisher über 4000 Triglycerid-Bestimmungen im Blutserum und anderen biologischen Flüssigkeiten sowie in Gewebeproben von Mensch und Tier durchgeführt. Ein Teil der Ergebnisse ẉird demnächst veröffentlicht (6).

1) Merck 5545 .

2) Riedel de Haën. 
Die Tabellen $3 \mathrm{a}$ und $3 \mathrm{~b}$ enthalten die Ergebnisse von Recovery-Versuchen; in Tabelle 4 sind die Resultate von Mehrfach-Bestimmungen (4 Untersucher) an verschiedenen Seren wiedergegeben. In allen Fällen ist die Wiederfindung gut, die Streuung gering.

Tab. $3 a$

Recovery-Versuch

Verschiedene Konzentrationen Olivenöl wurden nach Verseifung enzymatisch bestimmt

\begin{tabular}{ccc}
\hline $\begin{array}{c}\text { aus Einwaage } \\
\text { berechnet } \\
\text { mg/100 ml }\end{array}$ & $\begin{array}{c}\text { gefunden } \\
\mathrm{mg} / 100 \mathrm{ml}\end{array}$ & $\begin{array}{c}\text { Recovery } \\
\%\end{array}$ \\
\hline 100 & 99 & 99 \\
200 & 196 & 98 \\
400 & 394 & 98,5 \\
800 & 797 & 99.6 \\
1000 & 1001 & 100,1 \\
\hline
\end{tabular}

\section{Triglycerid-Bestimmung im Blutserum}

\section{Reagenzien}

\section{für die Verseifung}

a) 0,5N äthanol. $\mathrm{KOH}: 5,61 \mathrm{~g} \mathrm{KOH}$ (glycerinfrei, z. B. Riedel de Haën, Nr. 06103) werden in $200 \mathrm{ml}$ Äthanol p. a. gelöst oder 4,1 m/ Kalilauge (47proz., z. B. Merck 5545) wird mit Äthanol p. a. (absol.) auf $100 \mathrm{ml}$ aufgefüllt.

b) $0,1 \mathrm{Mr} \mathrm{MgSO}_{4}: 5,0 \mathrm{~g} \mathrm{MgSO}_{4} \cdot 7 \mathrm{H}_{2} \mathrm{O}$ werden in $200 \mathrm{~m} /$ Wasser gelöst.

fiir den Test (Endkonzentration im Ansatz)

$\begin{array}{ll}\text { Triäthanolamin } & (0,1 \mathrm{M}, \mathrm{pH} 7,6) \\ \mathrm{MgSO}_{4} \cdot 7 \mathrm{H}_{2} \mathrm{O} & (2 \mathrm{~mm}) \\ \mathrm{NADH} & \text { (etwa } 0,2 \mathrm{~mm}) \\ \text { PEP-Na } & (1 \mathrm{~mm}) \\ \text { ATP, Dinatriumsalz } & (0,2 \mathrm{~mm}) \\ \mathrm{LDH} & \text { (etwa 14U) } \\ \text { PK } & \text { (etwa } 2 \mathrm{U}) \\ \text { GK } & \text { (etwa } 1,3 \mathrm{U})\end{array}$

Herstellung der Lösungen für den enzy'matischen Glycerin-Test

a) Arbeitslösung I (Puffer TRA etwa 0,15M, pH 7,6): $2,62 \mathrm{~g}$ TRA-HCl und $0,035 \mathrm{~g} \mathrm{MgSO}_{4}$ werden in etwa $50 \mathrm{~m} / \mathrm{H}_{2} \mathrm{O}$ gelöst. Mit $\mathrm{NNaOH}$ auf $\mathrm{pH} \mathrm{7,6} \mathrm{einstellen} \mathrm{(pH-Meter).} \mathrm{Auffüllen} \mathrm{auf}$ $100 \mathrm{~m} /$ und darin $0,03 \mathrm{~g}$ NADH lösen.

b) Arbeitslösung II: 0,04 g PEP-Na und 0,03 g ATP-Na ${ }_{2} \mathrm{H}_{2}$ werden in $10 \mathrm{~m} / \mathrm{TRA}$-Puffer $(0,1 \mathrm{M}) \mathrm{pH} 7,6$ gelöst.

c) $\mathrm{LDH}(5 \mathrm{mg} / \mathrm{ml})$ und $\mathrm{PK}(2 \mathrm{mg} / \mathrm{ml})$ werden $1: 1$ gemischt.

d) GK $(1 \mathrm{mg} / \mathrm{m} /)$

Alle Lösungen für den Enzymtest werden im Kühlschrank aufbewahrt.

\section{Verseifung}

direkt

Man pipettiert $0,2 \mathrm{~m} /$ Serum und $0,5 \mathrm{~m} / 0,5 \mathrm{~N}$ äthanol. $\mathrm{KOH}$ in ein Schliffzentrifugenglas, verschließt es sorgfältig und verseift $30 \mathrm{Min}$ bei $70^{\circ}$ im Thermostaten. Nach Abkühlen des Hydrolysats setzt man $1,5 \mathrm{~m} / 0,1 \mathrm{M} \mathrm{MgSO}$-Lösung $\mathrm{zu}$, schüttelt gut durch und zentrifugiert $10 \mathrm{Min}$. Der klare Uberstand wird abgegossen und ist für den Test verwendbar. (Der Überstand ist bei Kühlschranktemperatur einige Tage haltbar.)

\section{Folch-Sperry-Extrakt}

In $5 \mathrm{~m} /$ Folch-Sperry-Lösung ${ }^{1}$ ) werden $0,2 \mathrm{~m} /$ Serum tropfenweise einpipettiert, gut geschüttelt und nach $20 \mathrm{Min}$. schnell durch ein grobporiges Filter filtriert. $2 \mathrm{~m} /$ der Extrakte werden in ein Schliffzentrifugenglas (14,5 er Schliff) gebracht und das Lösungsmittel verdampft. Man verseift den Rückstand mit $0,2 \mathrm{~m} l 0,5 \mathrm{M}$ äthanol. $\mathrm{KOH}$, wie oben beschrieben, gibt $0,5 \mathrm{~m} / \mathrm{MgSO}_{4}$-Lösung $\mathrm{zu}$ und nimmt ein Aliquot von $0,25-0,5 \mathrm{~m} l$ für den enzymatischen Test.

Testansatz

Man pipettiert $1,5 \mathrm{ml}$ Arbeitslösung I

$0,1 \mathrm{~m} l$ Arbeitslösung II

$0,5 \mathrm{~m} /$ Uberstand (oder weniger)

in eine Küvette,

rührt gut durch und liest die Extinktion bei $366 \mathrm{~nm}$ ab (kann auch entfallen). Dann setzt man $0,015 \mathrm{~m} / \mathrm{LDH} / \mathrm{PK}$-Gemisch zu, rührt durch und liest die Extinktion nach 10 und 15 Min. ab. Die Reaktion kommt zum Stillstand. Nun werden $0,015 \mathrm{~m} l \mathrm{GK}$ zugesetzt und wiederum nach 10 und $15 \mathrm{Min}$. abgelesen. Das $\Delta \mathrm{E}_{\mathrm{GK}}$ entspricht dem Glycerin-Gehalt und geht in die Berechnung ein. Ein Reagenzienblindwert wird bestimmt und in Abzug gebracht.

Hinveis:

Bei lipämischem Serum wird nur 0,1 oder $0,2 \mathrm{ml}$ des Üherstandes pipettiert und die entsprechende Menge Wasser dem Test zugesetzt.

Berechnung

$$
\frac{\left.\Delta \mathrm{E}_{\mathrm{korr} .} \cdot \mathrm{MG}^{2}\right) \cdot \mathrm{Küv} . \text { Vol. } \cdot \text { Verd. } \cdot 100}{3,3 \cdot 1000}=\mathrm{mg} / 100 \mathrm{ml}
$$

$$
\begin{array}{ll} 
& 0,2 \mathrm{ml} \text { Serum } \\
& +0,5 \mathrm{~m} l \mathrm{KOH} \\
& +1,5 \mathrm{~m} l \mathrm{MgSO}_{4} \text {-Lösung }
\end{array}
$$

$2,2 \mathrm{ml}$, davon $0,5 \mathrm{~m} / /$ Test.

1) Chloroform-Methanol 2:1 (v/v).

2) Als mittleres Molekulargewicht für Triglyceride in menschlichen und tierischen Körperflüssigkeiten (außer Milch) verwenden wir 850 .

\begin{tabular}{|c|c|c|c|c|c|}
\hline Serum + Ö-Emulsion & $\begin{array}{l}\text { Serum } \\
\text { Triglyceride } \\
\text { gefunden } \\
\mathrm{mg} / 100 \mathrm{ml}\end{array}$ & $\begin{array}{l}\text { Zusätze } \\
\text { Ol in } \mathrm{mg} / 100 \mathrm{ml}\end{array}$ & $\begin{array}{l}\text { Theorie } \\
\mathrm{mg} / 100 \mathrm{ml}\end{array}$ & $\begin{array}{l}\text { gefunden } \\
\mathrm{mg} / 100 \mathrm{ml}\end{array}$ & $\begin{array}{c}\text { Recovery } \\
\%\end{array}$ \\
\hline \multirow{2}{*}{$\begin{array}{l}\text { Serum }+10 \% \text { Emulsion } 1 \\
\text { Serum }+10 \% \text { Emulsion } 2 \\
\text { Serum }+2 \% \text { Emulsion } 1 \\
\text { Serum }+2 \% \text { Emulsion } 2\end{array}$} & 128 & 417 & 545 & \multirow{2}{*}{$\begin{array}{l}559 \\
527 \\
199 \\
207\end{array}$} & \multirow{2}{*}{$\begin{array}{r}102,5 \\
96,7 \\
94,3 \\
98,0\end{array}$} \\
\hline & 128 & 83 & 211 & & \\
\hline
\end{tabular}

Tab. $3 b$

Recovery-Versuch

Serum wurde mit einer etwa 5proz. Olivenöl-Emulsion gemischt und auf Triglyceride untersucht

\begin{tabular}{|c|c|c|c|c|c|c|c|c|c|c|}
\hline \multirow[b]{2}{*}{ Serum } & \multirow[b]{2}{*}{ a } & \multicolumn{3}{|c|}{ Direkthydrolyse, modifiziert } & \multirow[b]{2}{*}{$\begin{array}{l}\text { Mittelwert } \\
\mathrm{mg} / 100 \mathrm{ml}\end{array}$} & \multicolumn{5}{|c|}{ FOLCH-SPERRY-Extrakt } \\
\hline & & $b^{m g}$ & $\begin{array}{c}\mathrm{ml} \\
\mathrm{c}\end{array}$ & d & & a & b & $\underset{\mathrm{c}}{\mathrm{m} l}$ & d & $\begin{array}{l}\text { Mittelwert } \\
\mathrm{mg} / 100 \mathrm{ml}\end{array}$ \\
\hline $\begin{array}{l}1 \\
2 \\
3 \\
4\end{array}$ & $\begin{array}{r}121 \\
98 \\
782 \\
927\end{array}$ & $\begin{array}{r}118 \\
95 \\
775 \\
904\end{array}$ & $\begin{array}{r}121 \\
98 \\
782 \\
939\end{array}$ & $\begin{array}{r}118 \\
95 \\
779 \\
898\end{array}$ & $\begin{array}{r}119,5 s= \pm 1,81 \\
96,5 s= \pm 1,81 \\
779,5 s= \pm 3,36 \\
917,0 s= \pm 19,3\end{array}$ & $\begin{array}{r}92 \\
920\end{array}$ & $\begin{array}{r}93 \\
901\end{array}$ & $\begin{array}{r}94 \\
895\end{array}$ & $\begin{array}{r}93 \\
917\end{array}$ & $\begin{aligned} 93,0 \mathrm{~s} & = \pm 0,81 \\
908,2 \mathrm{~s} & = \pm 15,5\end{aligned}$ \\
\hline
\end{tabular}

Tab. 4

Fehlerberechnung an 4 Humanserumproben nach Direkthydrolyse, modifiziert bzw. im FoLCH-SPERRY-Extrakt 


\begin{tabular}{lcc}
\hline & Leerwert & Probe \\
\hline 0 Min. & 0,916 & 0,940 \\
LDH/PK & & \\
10 Min. & 0,887 & 0,874 \\
15 Min. & 0,885 & 0,872 \\
GK & & \\
25 Min. & 0,879 & 0,759 \\
30 Min. & 0,877 & 0,757 \\
\hline $\mathrm{E}_{\text {Glycerin }}$ & 0,008 & 0,115 \\
$\Delta \mathrm{E}_{\text {korrig. }}$ & & 0,107 \\
\hline
\end{tabular}

\section{Literatur}

1. Wieland, O., Biochem. Z. 329, 313 (1957). - 2. KReutz, F., Klin. Wschr. 40, 362 (1962). - 3. Eggstein, M. und F. Kreutz, Klin. Wschr. 44, 262 (1966). - 4. Sperri, W. M., Lipid Analysis. in: Methods of biochem. Analysis 2, 83, Interscience Publ., New York-London (1955). - FoLCH, J., J. biol. Chemistry 226, 497 (1957); siehe hierzu auch Zöllner, N. und D. EBERHAGEN, Untersuchung und Bestimmung der Lipoide im Blut, S. 36, Springer Berlin (1965). - 5. Schmidt, F. H. und K. v. DaHL, Klin. Wschr. im Druck. - 6. Schmidt, F. H., K. v. Dafil, W. SchNell und F. WILLIG, in Vorbereitung.

Dr. Felix H. Schmidt 68 Mannheim-Waldhof Sandhofer Str. 112-132

\title{
Eine fluorometrische Methode zur Testosteron-Bestimmung im Harn
}

\author{
Von V. Graef, P. Jobst ${ }^{1}$ ) und HJ. Staudinger \\ Aus dem Physiologisch-Chemischen Institut der Universität Gießen (Direktor: Prof. Dr. Hj. Staudinger)
}

(Eingegangen am 31. Januar 1968)

Es wird eine neue Methode zur Bestimmung von Testosteron im Menschen-Harn beschrieben. Nach enzymatischer Hydrolyse und Solvolyse wird der Harnextrakt an einer Säule aus Aluminiumoxid gereinigt. Testosteron wird von Epitestosteron und anderen $\Delta^{4}-3-K e t o-$ steroiden durch Dünnschichtchromatographie getrennt. Es wird durch die empfindliche Fluoreszenz-Reaktion auf Lithiumhydroxid quantitativ bestimmt. Die Methode ist schnell auszuführen und erfordert kleine Harnmengen ( $4 \mathrm{~m} /$ für Männer und $10 \mathrm{~m} /$ für Frauen).

A new method is reported for the determination of testosterone in human urine. After enzymatic hydrolysis and solvolysis the urine extract is purified on a column of aluminia. Testosterone is separated from epitestosterone and other $\Delta^{4}-3$-ketosteroids by thin-layer chromatography. It is measured by the sensitive fluorescence reaction on lithium hydroxide. The method is rapid and requires small urine samples $(4 \mathrm{~m} /$ for men and $10 \mathrm{~m} /$ for women).

Seitdem Schubert (1) 1960 erstmalig Testosteron aus menschlichem Harn isoliert und bestimmt hat, sind auch Methoden zur Bestimmung von Testosteron im Harn veröffentlicht worden. $\mathrm{Da}$ die Testosteron-Ausscheidung mit der Testosteron-Produktion korreliert, ist es sinnvoll, Testosteron im Harn zu bestimmen (2). Trotz Anwendung der verschiedensten Reinigungsverfahren gelang es nur wenigen Autoren (3-13), Testosteron von seinem 17 $\alpha$-Epimeren, Epitestosteron, sauber $z u$ trennen. Aus den Untersuchungen von De Nicola (7), Schubert (14) und Korenman (15) geht hervor, daß die ausgeschiedenen Mengen von Testosteron und Epitestosteron in etwa derselben Größenordnung liegen. Da abex Epitestosteron, verglichen mit Testosteron, nur eine ganz geringe androgene Aktivität besitzt, ist für eine genaue Bestimmung des Testosterons die Trennung der beiden Epimeren notwendig. Für die Endbestimmung bedienen sich einige Autoren der Gaschromatographie, andere benutzen isotopenmarkiertes Testosteron. Diese Methoden erfordern also besondere Apparate, die nicht überall verfügbar sind. Andere Methoden, bei denen das Testosteron bzw. sein Oxydationsprodukt colorimetrisch oder fluorometrisch bestimmt wird, sind oft nicht sehr empfindlich und erfordern daher den Einsatz größerer Harnmengen. Diẹ notwendigen Reinigungsverfahren sind meist sehr langwierig.

1) Stipendiat der Alexander-von-Humboldt-Stiftung.
Nach der von uns beschriebenen Methode läßt sich Testosteron von Epitestosteron durch Dünnschichtchromatographie trennen. Bei einer längeren Laufzeit des Dünnschichtchromatogramms kann man auch Epitestosteron von anderen störenden Steroiden trennen und für sich bestimmen. Zur quantitativen Bestimmung benutzen wir die Fluoreszenzmessung auf Lithiumhydroxid-Preßlingen (16). $\Delta^{4}$-3-Ketosteroide geben auf der Oberfläche eines Lithiumhydroxid-Preßlings eine spezifische und sehr empfindliche Fluoreszenz (17). Diese Reaktion haben wir bereits zur Bestimmung des Aldosterons im Harn verwendet (18).

\section{Methodik}

\section{Reagenzien}

Methanol p. a. (Fa. Riedel-de Haën) wird über eine Füllkörperkolonne destilliert.

Äthylacetat für Chromatographie (Fa. Riedel-de Haën), Chloroform p. a. (Fa. E. Merck, Darmstadt)

$\beta$-Glucuronidase/Arylsulfatase aus Helix pomatia (Fa. C. F. Boehringer, Mannheim)

Aluminiumoxid: neutral, Akt. II (Fa. Gebr. Giulini, Ludvigshafen)

Aluminiumoxid $\mathrm{GF}_{254}$ für die Dünnschichtchromatographie (Fa. E. Merck, Darmstadt)

Testosteron (Fa. E. Merck, Darmstadt)

Methylenchlorid: 2/ Methylenchlorid werden mit $200 \mathrm{~m} /$ 0,1proz. Kaliumpermanganat-Lösung, mit $200 \mathrm{~m} / \mathrm{N} \mathrm{NaOH}$ und dreimal mit je $400 \mathrm{~m} /$ Wasser ausgeschüttelt. Nach 24stdg. Trocknen über wasserfreiem Calciumchlorid wird das Lösungsmittel destilliert. 